\section{FLOW BEHAVIOUR OF OIL BLOB THROUGH A CAPILLARY TUBE CONSTRICTION DURING PULSED INJECTION}

\author{
Shiferaw Regassa Jufara*, Tarea M Al-Shamia, Ulugbek Djuraeva, \\ Berihun Mamo Negasha, Mohammed Mahbubur Rahmanb
}
aDepartment of Petroleum Engineering Universiti Teknologi PETRONAS, 32610 Seri Iskandar, Perak Darul Ridzuan, Malaysia bDepartment of Petroleum and Mineral Resources Engineering Old Academic Building (OAB), BUET, Dhaka 1000, Bangladesh

Article history

Received

16 November 2017

Received in revised form

19 July 2018

Accepted

1 August 2018

Published online

15 December 2018

*Corresponding author Shiferaw.jufar@utp.edu.my

\begin{abstract}
A numerical simulation of flow of oil blob through a capillary tube constriction is presented. The simulation was run in a 2D axisymmetric model. Water is injected at the inlet to mobilize oil blob placed near the capillary tube constriction. Transient flow images were used to understand the flow evolution process. Results from the study show that pulsed injection effectively assisted to squeeze out the oil blob through the capillary tube constriction with shorter time compared to continuous injection. Pulsed injection reduced the time required for the first droplet to cross the capillary tube constriction by about 3 folds compared to continuous injection. In addition, the droplet that crossed the constriction is larger when the flow was pulsed. In both cases, there is a reverse flow in the opposite direction of the injection. However, the severity of the reverse flow is stronger in the case of continuous injection. Immediately downstream the constriction, there is an adverse pressure gradient zone during continuous injection which limits the mobility of droplet that crossed the constriction. However, in the case of pulsed injection, there is a favorable pressure gradient zone immediately downstream the constriction. This zone expedites mobility of droplets that cross the constriction by transporting them further downstream through suction effect. Apparently, pulsed injection eases off the adverse pressure gradient and allowed more volume of oil to pass through the constriction. Within about two periods of pulsation, $84 \%$ of original oil placed at the beginning crossed the constriction compared to only $35 \%$ in the case of continuous injection. Even though the same amount of water was injected in both cases, pulsed injection clearly altered the flow behavior. The observation from this study may be extended to more complex flows in order to tailor the method for certain specific applications, such as flow of residual oil through a reservoir.
\end{abstract}

Keywords: Flow pulsation, Capillary tube flow, Flow excitation, Oil mobilization, Enhanced Oil Recovery

(C) 2019 Penerbit UTM Press. All rights reserved

\subsection{INTRODUCTION}

Flow of immiscible fluid through a constriction has several applications in many engineering field. Of particular interest is flow of oil through a reservoir. Oil and gas flows through a porous media where viscous, gravity and capillary forces are in competition. At the end of secondary recovery, the typical oil recovery factor from mature fields around the world is only between $20 \%$ and $40 \%$ [1]. Some of the remaining hydrocarbon can be recovered with the application of enhanced oil recovery (EOR) techniques developed over the last few decades [25]. In most cases, the conventional EOR techniques, which involve gas injection, steam flooding and chemicals injection, suffer from certain limitations. In light of this, many researchers are working on improving various aspects of EOR methods, such as screening techniques [6, 7], improving sweep efficiency [8], and exploring alternative techniques, such as Electromagnetic heating and Seismic excitation [9-12]. In an effort to maximize recovery factor, new techniques commonly known as unconventional EOR are being investigated [13-16]. One of these techniques is based on elastic vibration stimulation, which has been reviewed by Beresnev and Johnson [17]. Increase in oil production due to seismic activity was first observed in Russia [17]. Some of the change in production was associated with large-scale structural displacement. However, it was observed that far away from the epicenter where the reservoir is subjected to low frequency and low 
amplitude mechanical vibration, increase in production was observed. Inspired by such natural phenomenon, many researchers investigated the application of mechanical vibration as a tool for EOR application. Although the technology showed potential for practical applications, tests in some fields show mixed or inconclusive results [11, 18]. Several mechanisms have been proposed to explain how this technique results in improved recovery. Droplet percolation, viscosity reduction and unilateral movement due to low frequency perturbation are some of the mechanisms proposed in literatures. In addition, several theories related to effects of gravitational force and capillary pressure, such as change in wettability, alteration in relative permeability, coalescence and dispersion of the oil phase and reduction in viscosity were proposed. However, there is no general agreement between the theories that explain the effect of seismic excitation on fluid flow in a reservoir. As such, the key mechanism for increased production is not yet known. Apparently, clear understanding of the seismic wave stimulation mechanism is work in progress. The lack of full understanding of the physics of the technique hinders full practical application and makes performance prediction and project design very difficult and unreliable. At present, however, researchers focus on understanding the fundamentals of the technique to exploit full potential of the technology.

In this paper, our objective is to study flow characteristics of continuous and pulsed injections on their performance to squeeze oil blob through a capillary tube constriction. The model used mimics flow through a pore throat in a reservoir. The study was conducted numerically using a commercial software. The simplified model we used in this study represents a single pore throat in a porous media. Several researchers employed experimental and numerical techniques using similar micro-model in order to investigate various aspects microfluidics systems [19, 20]. Apparently, it is difficult to build all aspects of flow through a reservoir into such simplified model. However, the result obtained should be able to highlight basic flow characteristics in order to understand the underlying flow interactions.

\subsection{METHODOLOGY}

The Numerical simulation study presented in this paper is restricted to steady and laminar flow of an incompressible, Newtonian fluid through a rigid tube that has a constriction downstream the inlet. Apparently, such assumptions do not capture flow of hydrocarbon through a porous media. The primary objective in this study is to investigate how the flow behavior changes when the displacing fluid is injected in a continuously or in a pulsed manner.

The numerical study was conducted using FLUENT software. The volume of fluid (VOF) multiphase model was used in order to investigate the flow behavior.
Since there are two immiscible fluids involved, the VOF model was employed. The 2D axisymmetric capillary tube model shown in Figure 1 has a length of $5 \mathrm{~mm}$ and inlet width of $0.5 \mathrm{~mm}$. The center of the constriction with radius $0.186 \mathrm{~mm}$ is at $1.5 \mathrm{~mm}$ from the inlet. A circular oil droplet with radius $0.23 \mathrm{~mm}$ is placed $1 \mathrm{~mm}$ from the inlet. This location is in the fully developed flow zone and will be discussed later in the results section. To mimic the immiscible displacement process, the fluid injected at the inlet is water. In this study, water is the wetting fluid. In petroleum engineering, displacing non-wetting fluid with wetting fluid is commonly referred to as imbibition process.

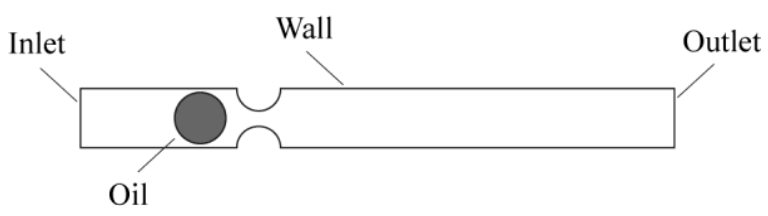

Figure 1 Geometry of the 2D axisymmetric model

The geometry was meshed in ANSYS workbench. To capture the large velocity gradient near the wall, inflation layer meshing was applied. To reduce the number of computations, the tube was modeled in 2D axisymmetric geometry. The mesh was imported to Fluent to perform the calculations and post processing. The boundary conditions are velocity inlet, pressure outlet and wall at the fluid solid interface. To capture wettability effect, the contact angle was 173, i.e. the wall is water wet. The density of water and oil are $1000 \mathrm{~kg} / \mathrm{m} 3$ and $720 \mathrm{~kg} / \mathrm{m} 3$, respectively. Water and oil viscosities are $0.001 \mathrm{~kg} / \mathrm{ms}$ and $0.00054 \mathrm{~kg} / \mathrm{ms}$, respectively. Water is primary phase and oil is secondary phase. In the phase interaction, wall adhesion was selected and the surface tension between water and oil was set to 27 dyn/cm. Fractional steps were used for pressurevelocity coupling. For pressure PRESTO, for momentum QUICK and for volume fraction geoReconstruct schemes were used. To keep the global courant number small, le-6 s time step was used [21]. In addition, to make sure results are independent of mesh and time steps, grid convergence and time step convergence tests were done. The simulations were run in first order implicit and non-iterative time advancement schemes.

In order to investigate the flow characteristics of continuous and pulsed injections, two different cases were simulated. In both cases, the outlet boundary was set to pressure outlet with 0 Pa gauge pressure. In the case of the continuous injection, water was injected at $5 \mathrm{~mm} / \mathrm{s}$ uniform velocity at the inlet. In the pulsed injection case, water was injected with a velocity that varied sinusoidal with amplitude \pm 0.75 the mean flow and frequency $20 \mathrm{~Hz}$. In both continuous and pulsed injections, the mass flow rate 
was the same. As such, the result obtained will be independent of mass flow rate.

\subsection{RESULTS AND DISCUSSION}

In order to place the oil droplet in the fully developed flow regime, we studied how velocity varies from the inlet along the axis of the capillary tube. The $x$-axis is aligned with the capillary tube centerline and the transverse axis is r. i.e., the center of the coordinate system, $x=0$ and $r=0$ is at the center of the inlet. Figure 2 shows the entrance velocity profile along the centerline of the capillary tube. At the entrance, the velocity profile is uniform with $5 \mathrm{~mm} / \mathrm{s}$ magnitude. The velocity boundary layer develops and eventually the profile becomes parabolic. The point where the velocity gradient along the $\mathrm{x}$-axis is zero marks the entrance length. In this case the entrance length is $0.48 \mathrm{~mm}$ and the maximum centerline velocity is 9.96 $\mathrm{mm} / \mathrm{s}$. The oil droplet is placed $1 \mathrm{~mm}$ from the entrance and it is in the fully developed flow zone. As such, longitudinal velocity gradient do not affect the oil blob.

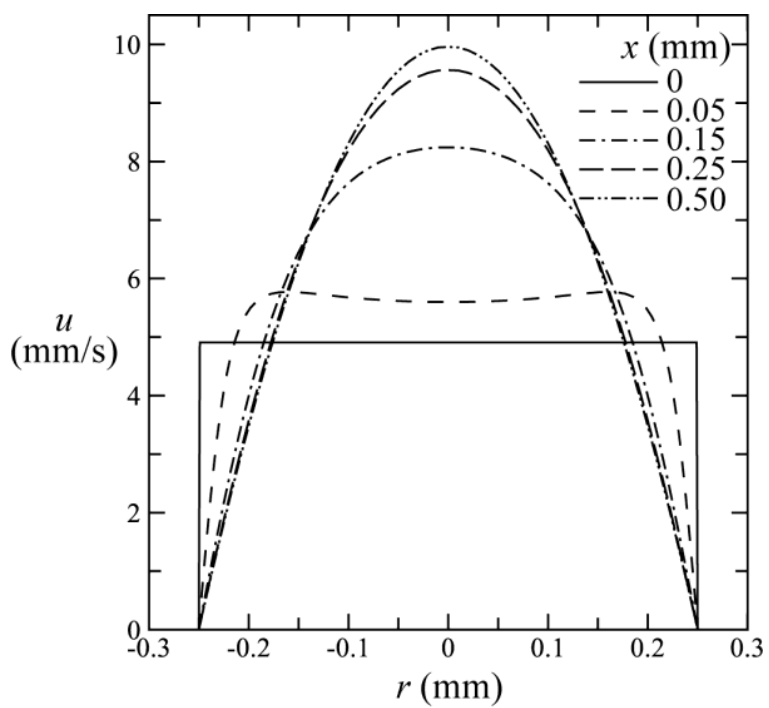

Figure 2 Velocity profile along the centerline of the capillary tube

Figure 3 shows phase volume fraction of the water and the oil phases during continuous injection. In the phase volume fraction, blue color is $100 \%$ oil and red color is $100 \%$ water. The flow evolution presented in Figure 3 shows the flow interaction during the first $0.090 \mathrm{~s}$ after the onset of injection. Figure $3 \mathrm{a}$ shows the beginning of the injection. At $t=0.004 \mathrm{~s}$, as shown in Figure 3b, small oil droplet breaks off from the undisturbed droplet at the beginning and arrived at the constriction. The small droplet sits at the narrowest point of the constriction until more droplets joined. Finally, the first droplet crossed the constriction around $0.012 \mathrm{~s}$. After the first small droplet crossed, lump of oil shown in Figure $3 \mathrm{c}$ blocked the constriction and small droplets snap off from the trailing edge and moved towards the inlet due to reverse flow as a result of the blockage. The injected water flows around the lump of oil and becomes a continuous phase again. At the same time, small droplets come off the leading edge of the oil blob and more oil crossed the constriction. The blockage, reverse flow and snap off at the trailing edge continues intermittently as shown in Figure 3c. More droplets coalesce and result in larger droplet around the inlet as shown in Figure $3 d$ and Figure $3 e$. Observation of the animations we recorded show that when the injected water impinge with the trailing edge of the oil droplet, it creates a recirculating vortex and the fluid that comes out of the recirculation flows back in the opposite direction of the injection. The recirculating water at the trailing edge causes low pressure zone which snaps off droplets from the oil blob and the droplets also move in the opposite direction to the injection due to the reverse flow described above.

(a) $t=0$

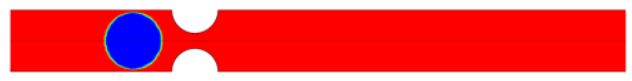

(b) $t=0.004 \mathrm{~s}$

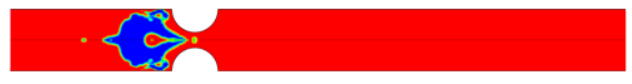

(c) $t=0.012 \mathrm{~s}$

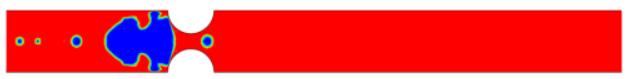

(d) $t=0.057 \mathrm{~s}$

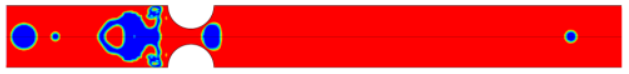

(e) $t=0.090 \mathrm{~s}$

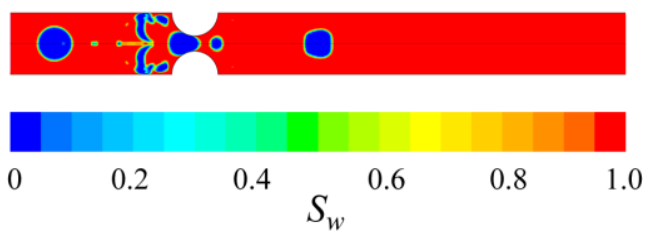

Figure 3 Flow evolution during continuous injection

In addition, the injected water that crosses through the constriction creates a recirculation region immediately downstream the constriction and creates a slightly high pressure zone, which is termed adverse pressure gradient region and is discussed below. For a significant period, the constriction sits 
between two high pressure regions. As such, it is difficult for the whole oil blob to cross through the constriction as a continuous phase. As a result, the original oil droplet breaks apart and coalesces continuously several times before it gets a chance to pass through the narrow constriction.

Based on this observation, we investigated the pulsed injection scenario in order to periodically ease off the amount of injected fluid to allow more oil droplets cross through the constriction as discussed below. The first small droplet shown in Figure $3 d$ crossed the length of the capillary tube in $0.077 \mathrm{~s}$. As can be seen from Figure $3 e$, the droplets shown in Figure $3 d$ that is carried in the opposite direction by the reverse flow starts to move forward after enough oil crossed the constriction and the severity of the reverse flow diminishes.

Figure 4 shows the flow evolution process during the pulsed injection. As discussed in the numerical model section, the mean flow was sinusoidally pulsed at $20 \mathrm{~Hz}$ frequency and +/-0.75 of the mean flow amplitude. It should be emphasized that the mass flow rate during continuous and pulsed injections during any full cycle of pulsation is the same.

(a) $t=0$

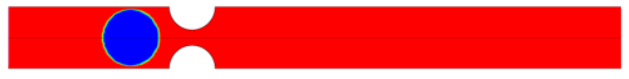

(b) $t=0.004 \mathrm{~s}$

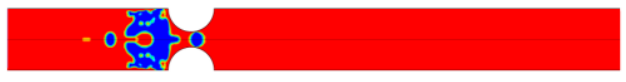

(c) $t=0.012 \mathrm{~s}$

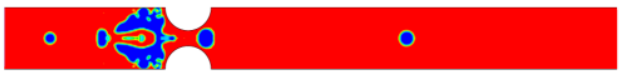

(d) $t=0.057 \mathrm{~s}$

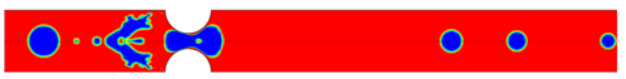

(e) $t=0.090 \mathrm{~s}$

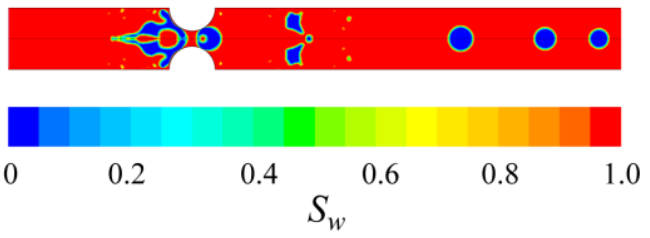

Figure 4 Flow evolution during the pulsed injection

Even though similar flow interactions discussed in the continuous injection case is seen during the pulsed injection, the strength of the reverse flow from the trailing edge of the oil blob is reduced. In addition, the two high pressure zones immediately downstream and upstream the constriction disappears for longer periods. That facilitated more droplets to cross through the constriction. The first droplet crossed through the constriction at about $0.004 \mathrm{~s}$ and crossed the length of the capillary tube at about $0.058 \mathrm{~s}$. Comparison of the continuous and the pulsed injections during the same time frame reveals how pulsation altered the flow characteristics significantly. The time for the first droplet to cross the constriction is shortened by 3 folds compared to continuous injection, which supports the results of experiments in core sample carried out by [22]. Even though the reverse flow still present during the pulsed injection as revealed by Figure $4 \mathrm{c}$ and Figure $4 \mathrm{~d}$, its strength is not as high as the continuous injection case. Figure $3 e$ and Figure $4 e$ shows the continuous injection and the pulsed injection at $t=0.090 \mathrm{~s}$, respectively. During continuous injection, large volume of oil was pushed by the reverse flow towards the inlet and sits near the entrance. However, when the flow is pulsed, significant volume of oil passed through the constriction and there was no oil left behind near the inlet due to the reverse flow as shown in Figure 4e. This observation is apparent in the animation we recorded.

\section{Continuous injection}

(a) $t=0.017 \mathrm{~s}$

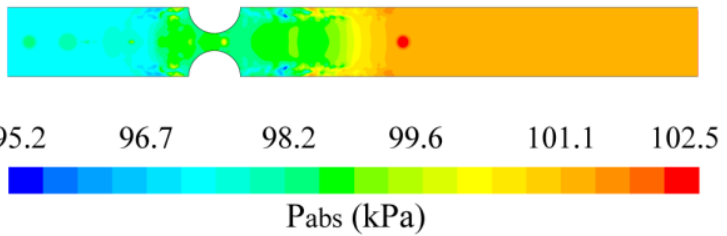

(b) $t=0.025 \mathrm{~s}$
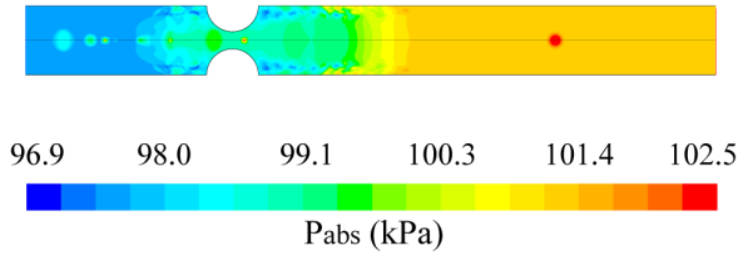

(c) $t=0.050 \mathrm{~s}$

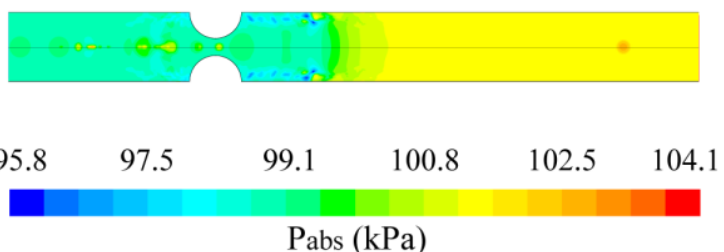

Figure 5 Contours of absolute pressure during continuous injection

Figure 5 shows absolute pressure contours during the continuous injection. The duration shown in Figure 5 is equal to one period of pulsation. In the case of continuous injection, upstream the constriction, absolute pressure is higher compared to downstream 
of the constriction. Such adverse pressure gradient restricts the passage of oil through the constriction. In particular, during the initial period of injection, there is high pressure zone at the constriction. As can be seen from Figure 3, such adverse pressure gradient is the cause of reverse flow in the opposite direction of injection along the centerline of the capillary tube. Apparently, pulsed injection should be able to periodically ease off the high pressure at the constriction and downstream the constriction and allow more oil to pass through the constriction. Near the wall of the capillary tube immediately downstream the constriction, there is pockets of low pressure zones due to flow recirculation. In some cases, such recirculation facilitates small droplets to cross the constriction.

Figure 6 shows absolute pressure contours during the pulsed injection. Similar to Figure 5, three frames within one period are presented. In contrast to continuous injection, the pressure contour drastically changed. Particularly, as can be seen from Figure 6b, the pressure contour is more favorable to push more oil blob through the constriction. At the end of the first period, i.e. Figure $6 \mathrm{c}$, the variation in pressure within the water phase is insignificant and the high pressure downstream the constriction disappeared.

\section{Pulsed injection}

(a) $t=0.017 \mathrm{~s}$
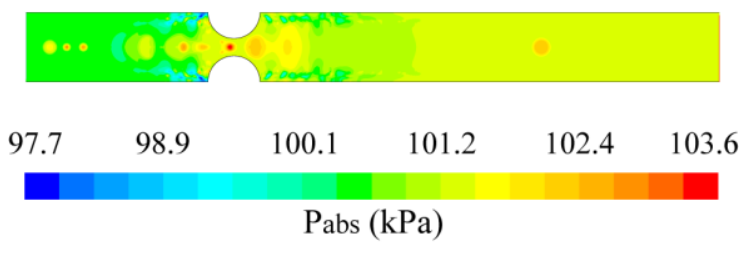

(b) $t=0.025 \mathrm{~s}$
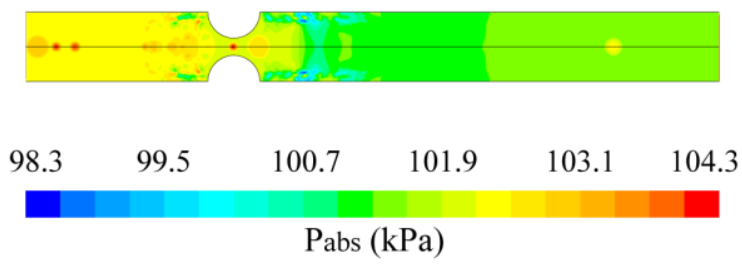

(c) $t=0.050 \mathrm{~s}$
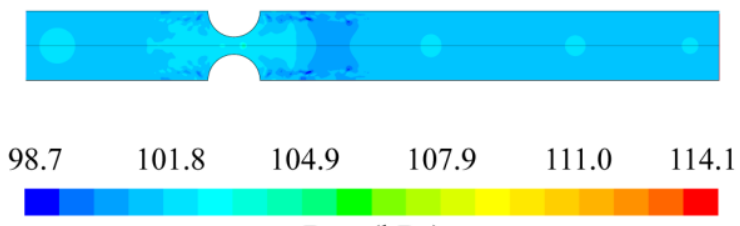

Pabs $(\mathrm{kPa})$

Figure 6 Contours of absolute pressure during pulsed injection

Comparing Figure $6 \mathrm{c}$ with phase volume fraction at the same instance, the 4 circular regions with slightly higher pressure are oil phases. In addition, the high-pressure area in and around the constriction are lump of oil in the process of crossing the constriction. Downstream the constriction, immediately after the lump of oil, there is a pocket of low-pressure region. This region has a suction effect on the lump of oil and allows large volume to cross the constriction. However, since this phenomenon occurs intermittently, before the continuous lump of oil crosses the constriction, another cycle of adverse pressure snaps off the oil and pushes it in the opposite direction of the flow. Compared to the continuous injection, however, the strength of the adverse pressure is low and more oil crosses within the same period. This observation is elaborated in detail in Figure 7 that is discussed below.

Figure 7 presents variation of absolute pressure along the centerline of the capillary tube during continuous injection. The pressure data has been filtered to suppress large picks due to phase change, i.e. oil droplets, along the centerline. Filtering the data did not change the profile as well as the value of the absolute pressure in the other sections of the capillary tube. Three times instances, the same as shown in Figure 5, are selected to present the absolute pressure profile. The bumps along the absolute pressure profile occur due to the presence of oil droplets at that particular position. In general, upstream the constriction, the absolute pressure gradient is positive, which means that during that particular period flow along the centerline is occurring in the opposite direction.

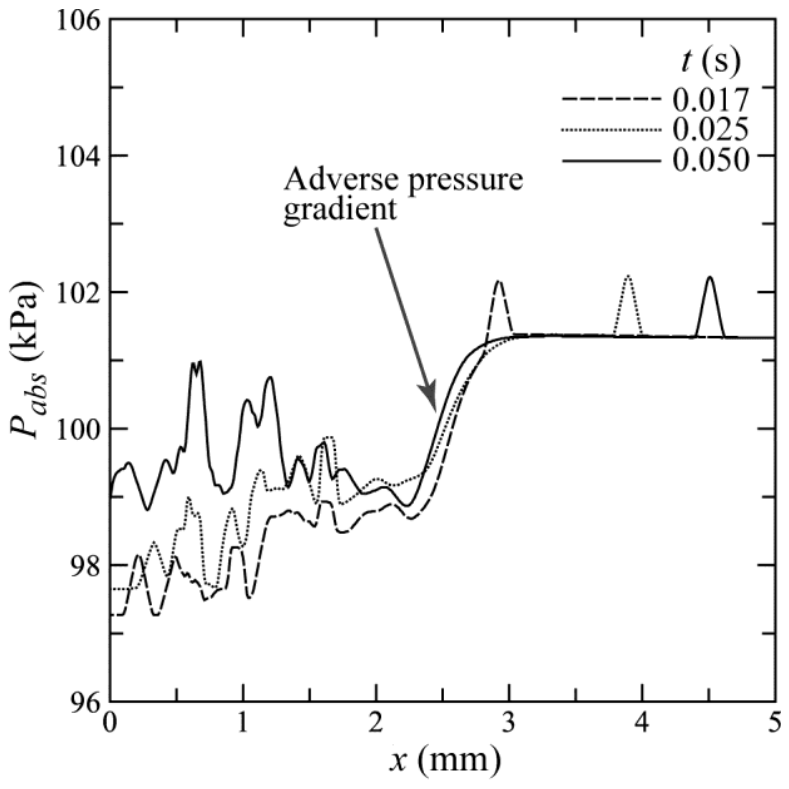

Figure 7 Absolute pressure profile along centerline of the capillary tube during continuous injection

The reverse flow gets worst when oil blob chocks the constriction due to the positive pressure gradient downstream the constriction, which is termed adverse pressure gradient region in Figure 7 . Immediately downstream the constriction, from time 
to time, the pocket of low-pressure recirculation zone near the wall expedites release of oil phase passing through the capillary tube constriction by entrainment process. These zones can be clearly seen in Figure $5 b$ and $c$. Once the flow crosses the adverse pressure gradient zone assisted by the lowpressure recirculation zones near the wall, eventually, it progresses downstream towards the exit.

Figure 8 shows the absolute pressure gradient along the capillary tube centerline during pulsed injection. Similar to what is presented in Figure 7, the data has been filtered to suppress sharp picks due to phase change along the centerline. Care has been taken to ensure the data in other section of the capillary tube remain unchanged. The bumps on the absolute pressure profiles are due to pressure discontinue because of phase change, i.e. oil droplets along the axis. At time $t=0.017 \mathrm{~s}$, the absolute pressure profile exhibits similar trend as the continuous injection. Eventually, the profile trend improves and allows mobilization of the oil phase. Particularly, at the end of the first cycle, the pressure change upstream and downstream the constriction stabilizes. In subsequent frames, similar trend shown at $\dagger=0.050$ s continuous.

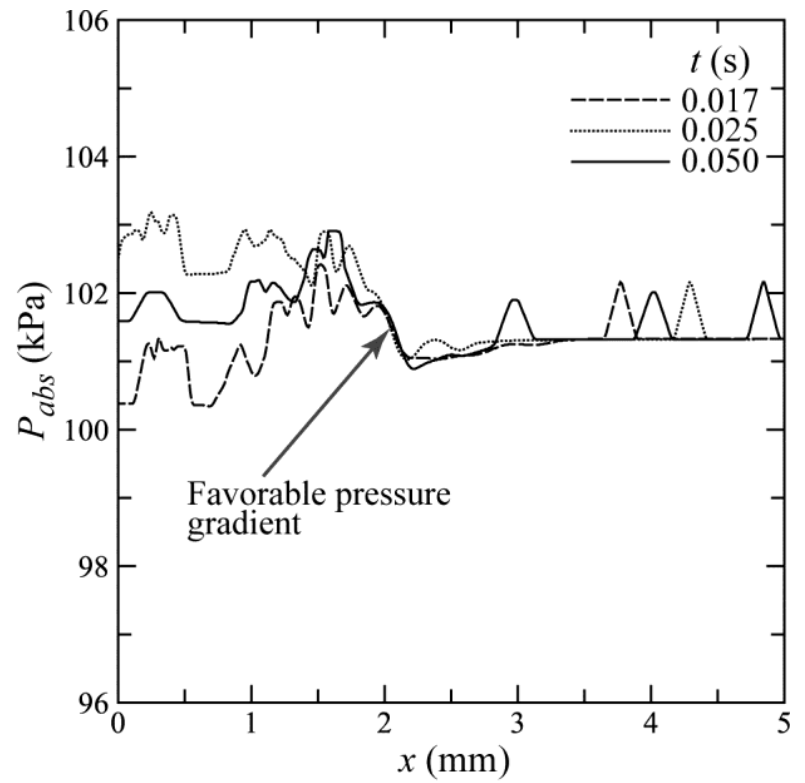

Figure 8 Absolute pressure profile along centerline of the capillary tube during pulsed injection

The significant difference during the pulsed injection is the occurrence of negative pressure gradient downstream the constriction, which is termed the favorable pressure gradient region as shown in Figure 8. Unlike the continuous injection case in which the oil droplet crossed the constriction stuck in the positive pressure gradient zone indicated as adverse pressure gradient in Figure 7 , the favorable pressure gradient zone creates suction effect and expedite transport of the oil droplets further downstream towards the exit. As such, it improves overall mobility of oil lumps. As evidenced by the pressure profile at $\dagger=0.017 \mathrm{~s}$ in Figure 8 , due to positive pressure gradient, there is a reverse flow. However, this situation improves with time. In the case of continuous injection, however, there is continuous reverse flow with some intermittent improvements due to interaction of oil lump with the constriction.

Figure 9 shows the fraction of oil that crossed the constriction at various times after injection. The secondary axis labeled $T$ is the corresponding period of pulsation. The fractions are calculated using an image analysis software by counting pixels that represent oil. Since the process is an immiscible displacement, oil is represented by blue color and water is represented by red color. All images are produced at the same resolution before the image analysis was carried out. The fraction of oil that crossed the center of the constriction is determined by adding the pixels that belong to oil at the particular period. The result from this analysis shows that significant fraction of oil crossed the constriction during the same period when the flow is pulsed. Within about two periods of pulsation, at $t=0.090$ s, $84 \%$ of the volume of oil at the beginning passed through the constriction during pulsed injection compared to only $35 \%$ in the case of continuous injection. Even though the mass flow rate is the same during any full period of excitation, about 2.5 times as much oil crossed the constriction when the flow is pulsed. In some instances, droplets that already crossed the constriction returns back due to the reverse flow. Such circumstances are prevalent during continuous injection.

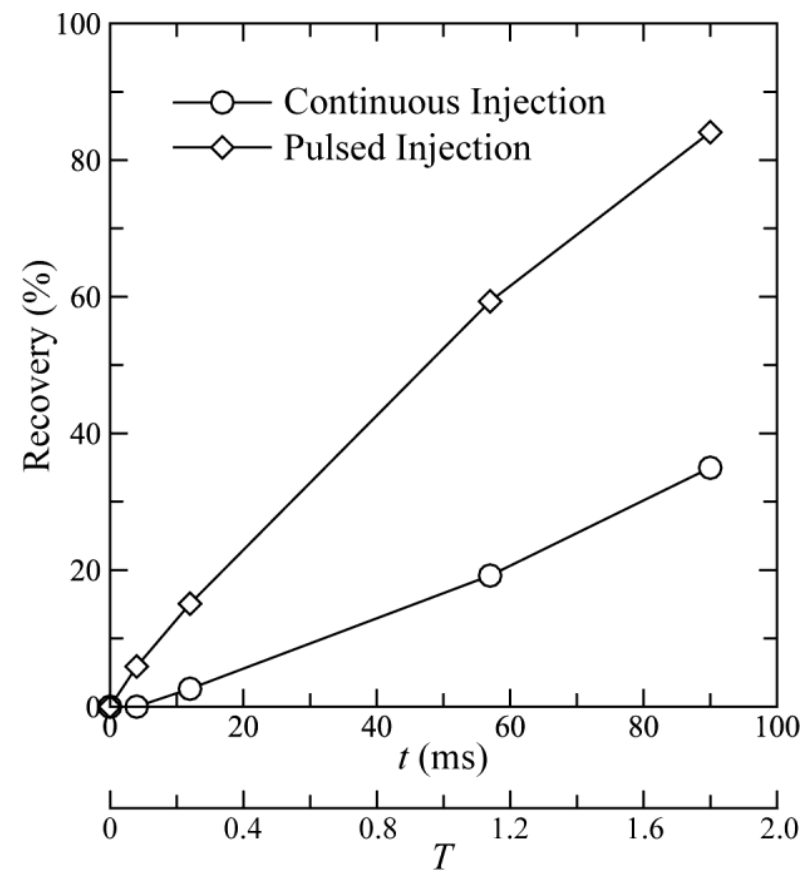

Figure 9 Recovery based on fraction of oil crossed the constriction 
The present study demonstrates how flow pulsation affects the characteristics of multiphase flow through a narrow constriction. Apparently, a simplified capillary tube model shown here cannot capture the full spectrum of flow dynamics through a porous media. In order to upscale and optimize the present concept for application in flow through a porous media, effects of injection parameters such as frequency, amplitude, waveform need to be studied in detail. In addition, fluid properties and fluid-solid interaction should be investigated in detail. Once the flow process is fully understood, the technique can be tailored for certain specific applications through upscaling of the controlling parameters.

\subsection{CONCLUSION}

The numerical study presented in this paper shows the flow characteristics through a capillary tube constriction during continuous and pulsed injections. The mass flow rate in both cases is the same. However, the flow characteristics are completely different. The understanding form such simplified flow models may be extended to flow through porous media as a means to mobilize residual oil. The result found here clearly demonstrates the benefit of flow pulsation.

In both continuous and pulsed injections, there is a reverse flow in the opposite direction of the injection. However, the severity of the reverse flow is stronger during continuous injection. In some cases of continuous injection, droplets that already crossed the constriction flow back upstream the constriction due to the reverse flow. Additionally, pulsed injection significantly reduced the time required for the first droplet to cross the constriction. During pulsed injection, the time for the first droplet to cross the constriction is reduced by three folds compared to continuous injection.

Immediately upstream the constriction there is an adverse pressure gradient zone in the case of continuous injection, and there is a favorable pressure gradient zone in the case of pulsed injection. While the adverse pressure gradient worsens the reverse flow, the favorable pressure gradient expedites the transport of droplets that cross the constriction further downstream towards the exit. In addition, the mechanism of oil transport in the case of continuous injection is through the entrainment action of the recirculation zones near the wall immediately downstream the constriction. In the case of pulsed injection, in addition to the entrainment action, the favorable pressure gradient enhances transport of more oil droplets. Within about two periods of pulsation, $84 \%$ of oil originally in place crossed the constriction compared to only $35 \%$ in the case of continuous injection.

\section{Acknowledgement}

This research is fully supported by Projects under grant No. 0153AA-C94 and 0153AA-E77. The authors fully acknowledged Universiti Teknologi PETRONAS for the approved fund which makes this important research viable and effective.

\section{References}

[1] S. R. Sandrea, I. 2007. Recovery Factors Leave Vast Target for EOR Technologies. Oil and Gas Journal. 105(4): 44-47.

[2] E. J. Manrique, C. P. Thomas, R. Ravikiran, M. Izadi Kamovei, M. Lantz, J. L. Romero, V. Alvarado. 2010. EOR: Current Status and Opportunities. Society of Petroleum Engineers.

[3] J. R. Hite, P. L. Bondor. 2004. Planning EOR Projects. Society of Petroleum Engineers.

[4] S. Afra, M. Tarrahi. 2015. Assisted EOR Screening Approach for CO2 Flooding with Bayesian Classification and Integrated Feature Selection Techniques. Carbon Management Technology Conference.

[5] A. Muggeridge, A. Cockin, K. Webb, H. Frampton, I. Collins, T. Moulds, et al. 2014. Recovery Rates, Enhanced Oil Recovery and Technological Limits. Philosophical Transactions. Series A, Mathematical, Physical, and Engineering Sciences. 372: 20120320-20120320.

[6] Al Adasani, B. Bai, 2011, Analysis of EOR Projects and Updated Screening Criteria. Journal of Petroleum Science and Engineering. 79(1-2): 10-24.

[7] R. B. C. Gharbi. 2000. An Expert System for Selecting and Designing EOR Processes. Journal of Petroleum Science and Engineering. 27(1-2): 33-47.

[8] R. Baker, C. Fong, C. Bowes, M. Toews. 2010. Understanding Volumetric Sweep Efficiency in SAGD Projects. Journal of Canadian Petroleum Technology. 49(01): 30-37.

[9] V. N. Nikolaevskiy, G. P. Lopukhov, L. Yizhu, M. J. Economides. 1996. Residual Oil Reservoir Recovery with Seismic Vibrations. SPE Production \& Facilities. 11 (2): 89-94.

[10] E. R. Abernethy. 1976. Production Increase of Heavy Oils by Electromagnetic Heating. Journal of Canadian Petroleum Technology. 15(03): 91-97.

[11] P. M. Roberts, I. B. Esipov, E. L. Majer. 2003. Elastic Wave Stimulation of Oil Reservoirs: Promising EOR Technology? The Leading Edge. 22(5): 448-453.

[12] Huh. 2006. Improved Oil Recovery by Seismic Vibration: A Preliminary Assessment of Possible Mechanisms. Society of Petroleum Engineers.

[13] O. Gurpinar. 2017. Technology Focus: EOR Performance and Modeling. Journal of Petroleum Technology. 69(01): 42-42.

[14] M. P. Rathmann, P. L. McGuire, B. H. Carlson. 2006. Unconventional EOR Program Increases Recovery in Mature WAG Patterns at Prudhoe Bay. Society of Petroleum Engineers.

[15] S. Rassenfoss. 2014. Carbon Dioxide May Offer an Unconventional EOR Option. Journal of Petroleum Technology. 66(2): 52-56.

[16] T. Hamida, T. Babadagli. 2006. Investigations on Capillary and Viscous Displacement under Ultrasonic Waves. Journal of Canadian Petroleum Technology. 45(02): 16-19.

[17] I. A. Beresnev, P. A. Johnson. 1994. Elastic-wave Stimulation of Oil Production: A Review of Methods and Results. GEOPHYSICS. 59(6): 1000-1017.

[18] P. M. Roberts, S. A. Kostrove, W. O. Wooden, E. L. Majer, T. M. Daley. 2001. Laboratory and Field Observations of Stress-wave Induced Changes in Oil Flow Behavior. Society of Exploration Geophysicists. SEG Technical Program Expanded Abstracts. 2001: 1682-1685. 
[19] S. Chingulpitak, S. Wongwises. 2010. Two-phase Flow Model of Refrigerants Flowing Through Helically Coiled Capillary Tubes. Applied Thermal Engineering. 30(14-15): 1927-1936.

[20] L. Yan, K. E. Thompson, K. T. Valsaraj. 2006. A Numerical Study on the Coalescence of Emulsion Droplets in a Constricted Capillary Tube. Journal of Colloid and Interface Science. 298(2): 832-844.
[21] A. Fluent. 2015. Ansys Fluent. Academic Research. Release 16(1): 14: 1336-1372.

[22] O. Kouznetsov, E. Simkin, G. Chilingar, and S. Katz. 1998. Improved Oil Recovery by Application of Vibro-energy to Waterflooded Sandstones. Journal of Petroleum Science and Engineering. 19(3): 191-200. 